\title{
Relationship between Coda-Q and Loaded Stress
}

\author{
Kyosuke OKAMOTO $^{1}$, Hitoshi MIKADA ${ }^{1}$, Tada-nori GOTO ${ }^{1}$ and Junichi TAKEKAWA ${ }^{1}$ \\ ${ }^{1}$ Dept. of Civil and Earth Res. Eng., Kyoto University
}

\begin{abstract}
A coda-wave is formed by superposed signals caused by scatterers such as cracks and medium inhomogeneities in the underground materials. For an inhomogeneous medium, it is natural to deal with stochastic methodologies to interpret seismic data. In the past, it is revealed that coda-Q, which is derived from the coda-wave has relationship with frequency of earthquakes ${ }^{1)}$. If the frequency of earthquakes is reflected the change of loaded stress, there is the relationship between the loaded stress and coda-Q. Our purpose is to reveal that relationship. We employ 2-D finite difference method to simulate scatter waves which is generated by cracks. When the stress is loaded to a simulation model, anisotropy of velocity is occurred along direction of the stress, and formation of the scatter waves is changed. Then value of coda-Q is changed. In this study, we find that coda-Q increases constantly according to the increasing stress and behavior of coda-Q has a tendency according to direction of the stress respectively (i.e. stress is loaded vertically or horizontally). In conclusion, we are able to estimate relative magnitude and the direction of the stress. It might lead a new method in underground monitoring.
\end{abstract}

\section{INTRODUCTION}

Coda-Q is obtained from the coda wave which is the last part of a seismic wave. Coda-Q is the parameter of attenuation. When the envelope of the coda wave is expressed as follows,

$$
u(t)=A_{0} e^{-\alpha\left(t-t_{0}\right)}
$$

coda-Q can be obtained by Equation (2).

$$
\alpha=\frac{\omega}{2 Q}
$$

where $A_{0}$ is initial amplitude, $t_{0}$ is initial time, $\alpha$ is an attenuation constant and $\omega$ is angular velocity.

The coda wave is summation of scatter waves which are reflected by cracks, boundaries and so on. Here, the coda wave becomes so complicated formation that it is difficult to analysis the wave using a deterministic model. However, the coda wave has much information because this wave is summation of the waves which come from everywhere. Generally, coda-Q is treated as the stochastic parameter. In the past, it is said that there are relationship between coda-Q and frequency of earthquakes $^{1)}$ or between coda-Q and b-value ${ }^{2)}$. They show coda-Q has relationship with the stochastic parameter. However in the past, there are few studies to find out the relationship between coda-Q and a specific physical parameter in underground. It is important to relate them by quantitative analysis. We try to relate them in this study.

Then, we employ 2D finite different method to simulate elastic wave propagation and analyze coda-Q which is changed by stress loaded to the simulation model. We discuss about relationship between coda-Q and the loaded stress.

\section{METHOD}

We employ 2D finite different method to simulate elastic wave propagation and scatter waves. We use staggered grid to discrete wave equation (3), equation of motion (4), (5) and stress-strain relations (6), (7), (8). Here, the accuracy of space and time are forth-order and second-order, respectively.

$$
\begin{gathered}
\rho \frac{\partial^{2} \theta}{\partial t^{2}}=(\lambda+2 \mu) \nabla^{2} \theta \\
\rho \frac{\partial^{2} u_{x}}{\partial t^{2}}=\frac{\partial \tau_{x x}}{\partial x}+\frac{\partial \tau_{x z}}{\partial z} \\
\rho \frac{\partial^{2} u_{z}}{\partial t^{2}}=\frac{\partial \tau_{x z}}{\partial x}+\frac{\partial \tau_{z z}}{\partial z} \\
\tau_{x x}=(\lambda+2 \mu) \frac{\partial u_{x}}{\partial z}+\lambda \frac{\partial u_{z}}{\partial z} \\
\tau_{z z}=(\lambda+2 \mu) \frac{\partial u_{z}}{\partial z}+\lambda \frac{\partial u_{x}}{\partial x} \\
\tau_{x z}=\mu\left(\frac{\partial u_{x}}{\partial z}+\frac{\partial u_{z}}{\partial x}\right)
\end{gathered}
$$

Fig. 1 shows the simulation model we use.₹ 


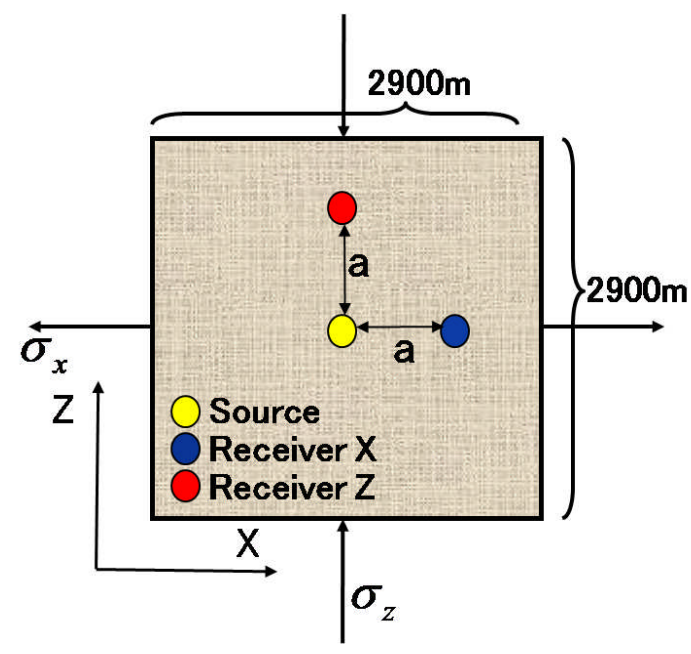

Fig. 1 simulation model

The scale of simulation model is $2900 \mathrm{~m} \times 2900 \mathrm{~m}$ which is expressed by $1000 \times 1000$ grids. The profiles of the medium are shown below. $\mathrm{V}_{\mathrm{p}}=5.8 \mathrm{~km} / \mathrm{sec}, \mathrm{V}_{\mathrm{S}}=3.2 \mathrm{~km} / \mathrm{sec}, \rho=3.3 \mathrm{~g} / \mathrm{cm}^{3}$. We put a source and two receivers in the simulation model like Fig. 1 respectively. We also put scatterers, which size is $1 \times 1$ grid in the model randomly.

Here pure shear stress is loaded to the model. Along the direction of $\mathrm{x}$-axis, compressive stress is loaded and the direction of z-axis, tensile stress is loaded. The magnitude of compressive stress and tensile stress is equal. We assume that the model is distorted and anisotropy of velocity is caused by loaded stress. Anisotropy of velocity is occurred by closure and generation of the cracks due to the loaded stress.

In this study, to reveal the relationship between coda-Q and loaded stress, coda-Q is repeatedly observed and analyzed during magnitude of the stress is increased from 5.0MPa to $50 \mathrm{MPa}$.

\section{RESULTS}

We compare the behavior of coda-Q in terms of (1) the location of the receiver, (2) distance between the source and the receivers and (3) distribution of aspect ratio.

\section{(1) The location of receiver}

With pure shear loaded, we observe coda-Q at two receivers respectively. Fig.2 shows that result.

Vertical axis is coda- $\mathrm{Q}^{-1}$, which is inverse of coda-Q and horizontal axis is the magnitude of the loaded stress. Coda- $Q^{-1}$ which is obtained by the receiver $\mathrm{Z}$ is always greater than coda- $\mathrm{Q}^{-1}$ which is obtained by the receiver $X$. This is due to closure of the cracks whose normal vector is toward to z-axis. By the closure, velocity along z-axis is increased and attenuation ratio is also increased.

\section{(2) Distance between the source and the receivers}

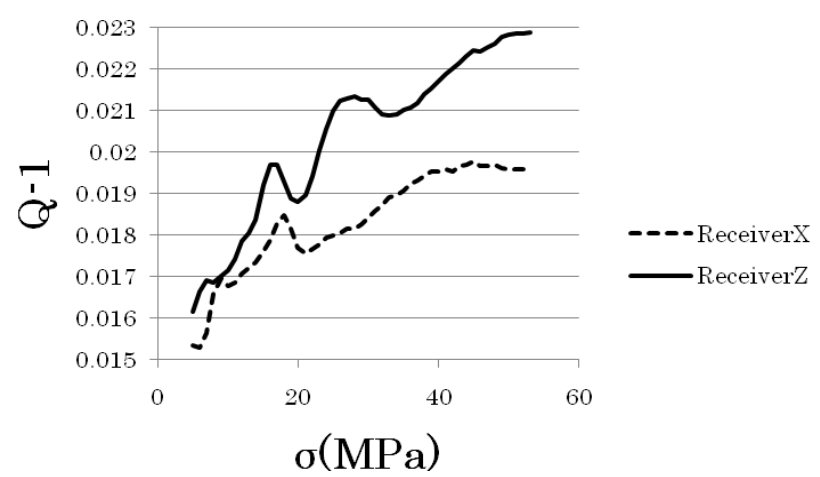

Fig. 2 Distance between the sauce and receivers is $638 \mathrm{~m}$.

Fig. 2 shows the result when the distance between the source and the receivers is $638 \mathrm{~m}$. Then we check the behavior of coda- $\mathrm{Q}^{-1}$ with the distance changed. Fig. 3 shows the result when the distance is changed.

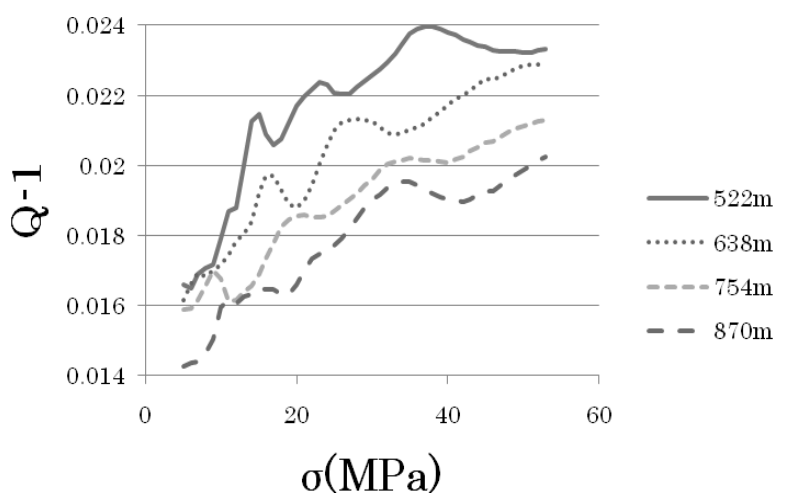

Fig. 3 Distance between the source and the receivers is changed.

As shown in this figure, we can see two tendencies. One is that the shorter the distance is, the grater coda- $\mathrm{Q}^{-1}$ becomes. The other is that the stronger the magnitude of the stress becomes, the grater coda- $\mathrm{Q}^{-1}$ becomes at every distance.

\section{(3) Distribution of aspect ratio}

Aspect ratio of all cracks is same when we discuss about (1) and (2). Then we assume the aspect ratio of the cracks has certain range. We consider two distributions of the aspect ratio. One is Pattern A; condition of $\alpha_{A}(n)$ is $0<\alpha_{A}(n)<5.0 \times 10^{-4}$. The other

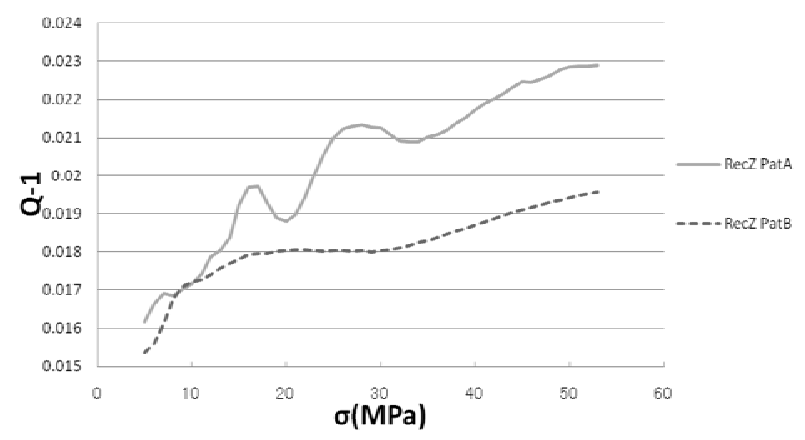

Fig. 4 aspect ratio is changed 
is Pattern B; condition of $\alpha_{B}(n)$ is $0<\alpha_{B}<2.5 \times 10^{-4}$. Fig. 4 shows the result when the distribution of aspect ratio is changed.

Coda- $\mathrm{Q}^{-1}$ derived from Pattern $\mathrm{A}$, which aspect ratio is greater than pattern $\mathrm{B}$ is always greater than the other. This is because that attenuation is grater when aspect ratio is bigger.

\section{CONSIDERATION}

In conclusion, we would like to propose that the loaded stress could be estimated (e.g. direction of the stress and relative magnitude of the stress) if we obtain the change of coda-Q varying with the loaded stress. It means that non-stochastic physical-state properties could be obtained from coda-wave and will lead to more clear deterministic model which is essential for engineering application of wave theories.

\section{REFFERENCE}

1) Aki, K., 2004, A Perspective on the Engineering Application of Seismology, The7th SEGJ International Symposium, Invited Paper

2) Hiramatsu, Y. , Hayashi, N. , Furumoto, N. , Katao, H., 2000, Temporal changesin coda $\mathrm{Q}^{-1}$ and $b$ value due to the static stress changes with the 1995 Hyogo-kenNanbu earthquake, JOURNAL OF GEOPHYSICAL RESERCH, VOL. 105, NO.B3, PAGES 6141-615 